\title{
How Do Room and Apparatus Cues Control Navigation in the Morris Water Task? Evidence for Distinct Contributions to a Movement Vector
}

\author{
Derek A. Hamilton and Katherine G. Akers \\ University of New Mexico
}

\author{
Michael P. Weisend \\ University of New Mexico and Mental Illness and Neuroscience \\ Discovery Institute
}

\author{
Robert J. Sutherland \\ University of Lethbridge
}

\begin{abstract}
The present study compared the relative influence of location and direction on navigation in the Morris water task. Rats were trained with a fixed hidden or cued platform, and probe trials were conducted with the pool repositioned such that the absolute spatial location of the platform was centered in the opposite quadrant of the pool. Rather than swimming to the platform location, rats swam in the direction that was reinforced during training, resulting in navigation to the relative location of the platform in the pool and search at the appropriate distance from the pool wall. Pool relocation tests revealed disruptions in cued navigation if the cued platform remained at the absolute location, whereas no disruption was observed if the platform remained at the relative location (same direction). The results indicate that direction holds greater influence than does location and further demonstrate that this observation is not altered by the amount of training or time on the platform. The authors propose that navigation in the water task involves a movement vector in which the distal cues and apparatus provide direction and distance information, respectively.
\end{abstract}

Keywords: spatial learning, cognitive mapping, hippocampus, water maze, cued navigation

Supplemental data: http://dx.doi.org/10.1037/0097-7403.33.2.100.supp

The processes by which animals navigate between spatial locations have been intensely studied for many years and have been the subject of considerable debate. Place navigation is a term commonly used to indicate that animals navigate to specific places (e.g., a goal location) on the basis of the fixed spatial relationship between the location of interest and conspicuous exteroceptive stimuli (e.g., distal visual cues). This ability was a central feature of Tolman's cognitive mapping theory (Tolman, 1948; Tolman, Ritchie, \& Kalish, 1946) and, later, the influential mapping theory of O'Keefe and Nadel (1978). Perhaps the most intensely debated topic within this literature concerns precisely what is learned when navigation to a specific place is reinforced in the presence of distal visual cues. Mapping theory argues that animals navigate to precise spatial locations on the basis of a learned representation of the environment that includes where reinforcement occurs. During the

Derek A. Hamilton, Department of Psychology and Department of Neurosciences, University of New Mexico; Katherine G. Akers, Department of Psychology, University of New Mexico; Michael P. Weisend, Department of Psychology, University of New Mexico and Mental Illness and Neuroscience Discovery Institute, Albuquerque, NM; Robert J. Sutherland, Canadian Centre for Behavioural Neuroscience, University of Lethbridge, Lethbridge, Alberta, Canada.

We thank Henrik Gemoll, Brandon Wesenberg, Katrina Barnes, Andrea Watts, and Sylwia Gutierrez for assistance with data collection and analysis. We also thank Elise McHugh for assistance in preparing the manuscript.

Correspondence concerning this article should be addressed to Derek A. Hamilton, MSC03 2220, Department of Psychology, University of New Mexico, Albuquerque, NM 87131. E-mail: dahamilt@unm.edu early years of this debate, several alternative explanations were considered, including the learning of specific motor responses (Blodgett \& McCutchan, 1947; Blodgett, McCutchan, \& Mathews, 1949), approach-avoidance tendencies (Hull, 1943), and movement in a particular direction in the environment (Blodgett et al., 1949; see also Olton, Becker, \& Handelmann, 1979).

Recently, the idea that animals navigate to goal locations by moving in a particular direction in the environment has received renewed interest (Skinner et al., 2003; Sutherland \& Hamilton, 2004). To contrast navigation to places with directional responding, Skinner et al. (2003) trained rats to navigate to a goal location in a square open-field environment in the presence of multiple distal visual cues. Reinforcement was always located in one corner of the open field and the apparatus was repeatedly moved between two locations during training. One group of rats was trained such that movement in a particular direction (to the east-most corner opposite the release point) was reinforced for both apparatus locations, whereas another group was trained such that navigation to the same place in the room was reinforced regardless of the apparatus location. In agreement with the findings of Blodgett et al. (1949), the results of Skinner et al.'s study clearly demonstrated that directional responding was learned much more quickly than was navigation to a precise location. A consistent finding across these studies is that navigation to places requires the most training to meet criterion, compared with other forms of responding, prompting these authors to conclude that the contribution of place information to maze navigation is negligible.

The broad goal of the present study is to examine the relative contributions of information about location and direction to navigation in the Morris water task (Morris, 1981, 1984). In this task, 
rats are trained to swim to a submerged (hidden) escape platform located in a fixed spatial location relative to a constellation of distal visual cues. Rats that have mastered this task take more or less direct trajectories to the platform from multiple release points and persist in searching at the platform location when it is removed during a probe trial (Morris, 1981; Sutherland \& Dyck, 1984). It is generally agreed that distal visual cues provide the critical source of control in this task; however, whether animals solve the task by navigating to a precise location or by directional responding is not clear. Because the apparatus and the platform remain in the same location in the room throughout training and testing, it is impossible to rule out directional responding as one way by which animals navigate to the platform in the fixed platform water task.

The present set of experiments follows an initial observation in our laboratory (Weisend et al., 1995, and discussed in Sutherland \& Hamilton, 2004) in which clear evidence for directional responding was observed in the Morris water task when the pool was relocated in the room. To our knowledge, a characterization of this phenomenon and the conditions under which it occurs in the Morris water task is not currently represented in the literature. For the present experiments, a Morris water task apparatus was constructed that allowed the pool to be positioned at either of two locations in the environment. Figure
1 shows the layout of the room, the two locations where the pool could be positioned, and the three locations where an escape platform could be placed. If an animal is trained to navigate to the escape platform at Location B and the pool is in Position 1, then moving the pool to Position 2 and removing the platform for a probe trial could result in several outcomes. The animal could navigate to the same, absolute spatial location (Location B) where the platform was located during training, which would support the hypothesis that rats navigate to specific locations. Alternatively, the animal could navigate in the direction of the platform location in the room (e.g., to the east), resulting in navigation to the relative location within the pool where the platform was located during training (Location C). Because the platform is always a fixed distance from the pool wall during training, directional responding could be followed by highly focused search at the relative location (Location $\mathrm{C}$ ) based, in part, on distance information provided by the apparatus. If this pattern is observed, then it would seem that directional responding and control by the apparatus would provide a reasonable alternative to the idea that animals navigate to and persist in searching at an absolute spatial location in the Morris water task. Related to this possibility, a secondary goal of the present experiments is to highlight the utility of explaining navigation
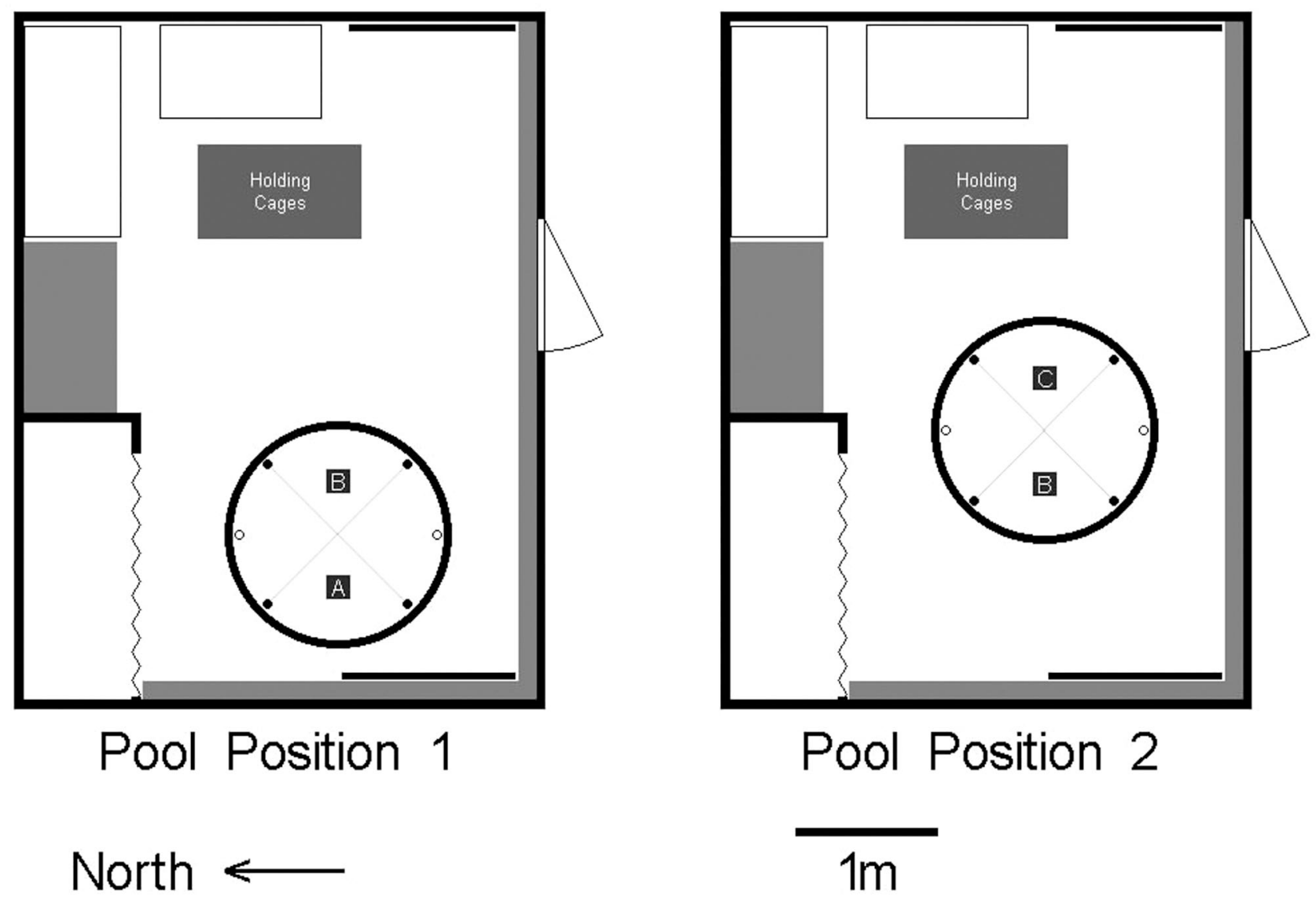

Figure 1. Layout of the testing room showing the room geometry and location of prominent visual cues (gray or black rectangles). The pool was located in one of two positions that were separated by $75 \mathrm{~cm}$ (the pool radius). Three platform locations labeled A, B, and C were used. Note that Platform Location B represents the same absolute spatial location within the room reference frame for both pool positions. The dark circles inside the pool mark the four release points used during hidden platform training and the open circles represent the two release points sampled for the no-platform probe trial. 
during a single Morris water task trial in terms of multiple, distinct sources of control.

\section{Experiment 1}

Experiment 1 was designed to evaluate the effects of moving the pool in the room on the direction of the swim trajectory and search behavior in animals that were trained to swim to an escape platform in a fixed location. Rats were given 36 hidden platform training trials over the course of 3 days, and the pool and platform location were fixed throughout training. All possible combinations of pool and platform location were trained for different groups of rats (see Figure 1). At the end of training, a probe trial was conducted in which the pool was either moved to another (untrained) position, such that the absolute spatial location and the relative spatial location were put into conflict, or remained in the same position used during training. Performance when the absolute location was no longer within the perimeter of the pool during the probe trial was also assessed. If rats learn to navigate to a particular spatial location on the basis of its fixed relationship to the distal visual cues, then moving the pool should result in a preference for navigation to the absolute spatial location. Mapping theories (O'Keefe \& Nadel, 1978; Tolman, 1948), which postulate that animals construct a map-like representation of space, would be able to explain this pattern of behavior, as would other explanations, such as perceptual matching (e.g., Wilkie \& Palfrey, 1987) in which an animal navigates to a particular place by attempting to match a perceptual memory of what the environment looked like from the goal location.

Alternatively, the control provided by distal cues in the Morris water task could be predominantly related to the direction of trajectories selected during navigation. For example, Sutherland, Chew, Baker, and Linggard (1987) and Hamilton, Driscoll, and Sutherland (2002) found that rats and humans, respectively, learn to take trajectories to the platform within a familiar range of views experienced during training. One way in which these findings can be conceptualized is that subjects learn to move in a particular direction by approaching and/or avoiding cues experienced while swimming to and locating the platform. If the results of Skinner et al.'s (2003) study generalize to the Morris water task, then moving the pool should result in execution of a trajectory based on the direction in the room that resulted in escape from the water during training. This type of trajectory selection could be combined with control by the pool wall such that an animal swims in a particular direction and then searches for the platform at the appropriate distance from the pool wall. If so, then animals should perform this type of response regardless of whether the absolute spatial location is directly in competition with the relative location or the absolute location is completely outside the perimeter of the apparatus.

\section{Method}

Subjects. Subjects were 32 male hooded Long-Evans rats (Rattus norvegicus; Charles River Laboratories, Wilmington, MA) that were approximately 90 days of age at the beginning of the experiment. The rats in Experiment 1 had served as subjects in a water task experiment conducted approximately 70 days earlier when the animals were 17-24 days of age. ${ }^{1}$ All animals were pair housed in plastic cages on a 12-hr light-dark cycle with food and water available ad libitum. Behavioral testing was performed during the light phase from approximately 1200-1300. All procedures for the studies reported here were approved by the Institutional Animal Care and Use Committee at the University of New Mexico.

Apparatus. The circular pool $(1.5 \mathrm{~m}$ diameter, $48 \mathrm{~cm}$ high $)$ was placed on a wooden frame (48 $\mathrm{cm}$ tall) that rested on appliance rollers, making it possible to easily move the pool when it was filled with water. The escape platform was constructed of plastic with a $16 \mathrm{~cm} \times 16 \mathrm{~cm}$ top surface and a height of $25 \mathrm{~cm}$. The pool was filled to a depth of $26 \mathrm{~cm}$ with cool water $\left(22{ }^{\circ} \mathrm{C}\right)$ that was made opaque by adding a small amount $(\sim 56.70 \mathrm{~g})$ of powdered white tempura paint. The testing room contained numerous distal visual cues, and the room walls formed a complex geometry (see Figure 1). Behavior was videotaped via an overhead camera and digital camcorder. The digital video was transferred to a Linux workstation for tracking and analysis.

Design and procedure. Experiment 1 was conducted in two phases, a training phase and a test phase. During the training phase, all rats were given 12 hidden platform trials during each of three daily sessions. During each daily session, rats were released three times from each of four possible release points around the perimeter of the pool (see Figure 1). The order of release points was selected randomly without replacement during each block of four trials. Rats were released facing the wall of the pool and were retrieved from the platform $5 \mathrm{~s}$ after mounting it. If a rat did not find the platform within $60 \mathrm{~s}$, it was retrieved and placed on the platform for $5 \mathrm{~s}$. Two pool positions were used, and there were three possible platform locations within the room (see Figure 1). An equal number of rats $(n=8)$ were randomly assigned to each of the four possible combinations of pool position and platform location (see Table 1). Once determined, the pool position and platform location remained fixed for each rat throughout training. For each training trial, we measured the latency to find the platform and computed each rat's mean latency for each trial block.

On completion of the final training trial on Day 3, a 30-s no-platform probe trial was conducted. Release points for the probe trial were selected at random from two points that were not used during the training phase (the north-most and south-most points around the perimeter of the pool; see Figure 1). Half of the animals for each combination of pool position and platform location were assigned to either a Shift condition $(n=16)$, where the pool was moved $75 \mathrm{~cm}$ (from the trained position to the other pool position), or to a No Shift condition $(n=16)$, in which the pool remained in the trained position. For half of the animals in the Shift condition, the pool was moved such that the absolute spatial location of the platform during training was still within the pool but was now located in the center of the opposite quadrant. Thus, the absolute spatial location of the platform within the room and the relative spatial location within the pool were placed into competition with one another. This condition is referred to as the Shift-Absolute versus Relative condition. For the remaining half

\footnotetext{
${ }^{1}$ Rats in this experiment were subjects in a study on development of place navigation in which each animal was given 24 hidden platform trials during one postnatal day (P17, P18, P19, P20, or P24). All training was performed in the same room as used in the present experiments; however, a different pool position and platform location were used. Only rats tested on P24 showed clear evidence of place learning, whereas rats tested on P17-P20 did not learn to take direct trajectories to the platform. Equal numbers of rats from each postnatal testing day were assigned to each of the conditions of Experiment 1.
} 
Table 1

Combinations of Training Pool Position, Training Platform Location, and Probe Trial Pool Position (See Figure 1) Used for Each Condition in Experiment 1

\begin{tabular}{|c|c|c|c|}
\hline Condition & $\begin{array}{c}\text { Training } \\
\text { Pool position }\end{array}$ & $\begin{array}{c}\text { Training } \\
\text { Platform location }\end{array}$ & $\begin{array}{c}\text { Probe trial } \\
\text { Pool position }\end{array}$ \\
\hline
\end{tabular}

No Shift

\begin{tabular}{llll} 
& 1 & A & 1 \\
& 1 & B & 1 \\
& 2 & B & 2 \\
Shift: Absolute & 2 & C & 2 \\
$\quad$ vs. Relative & & & \\
& 1 & B & 2 \\
Shift: Relative Only & 2 & B & 1 \\
& 1 & A & 2 \\
& 2 & C & 1 \\
\hline
\end{tabular}

Note. Each row represents a combination of pool positions and training platform location used for four animals.

of the animals in the Shift condition, the pool was moved such that the absolute spatial location of the platform was no longer within the pool but was approximately $37 \mathrm{~cm}$ beyond the pool wall. This condition, referred to as the Shift-Relative Only condition, allows for an assessment of navigation to and persistence at the relative spatial location during the probe trial without direct competition from the absolute spatial location (i.e., when it is not possible to reach the absolute spatial location within the room reference frame). If rats in the Shift-Absolute versus Relative condition distribute their time evenly between the absolute and relative spatial locations, then they should spend less time in the relative spatial location compared with rats in the Shift-Relative Only condition. That is, the cues that control navigation to the absolute spatial location should afford less effective competition in the Shift-Relative Only condition compared with the Shift-Absolute versus Relative condition. Further, if animals in the Shift-Absolute versus Relative condition prefer the relative location, then navigation to and persistence at the absolute location in this condition can be directly compared with the same measures for the quadrant opposite the absolute location in the No Shift condition and the relative location in the Shift-Relative Only condition. This provides information about the degree to which animals in the ShiftAbsolute versus Relative condition have learned to navigate to the absolute spatial location during training.

Four probe trial dependent measures were taken for each of two critical locations in the pool. For each group, there were two critical locations that were the same size as the platform surface. For the Shift-Absolute versus Relative condition, the two critical locations were the absolute platform location and the relative platform location within the pool (which was in the quadrant opposite the absolute location). For the No Shift condition, the critical locations were the absolute platform location and a comparable location in the opposite quadrant of the pool. For the Shift-Relative Only condition, the two critical locations were the relative spatial location of the platform within the pool and a comparable location in the opposite quadrant. The opposite location for the No Shift and Shift-Relative Only conditions was chosen as a critical location because the absolute and relative locations are in opposite quadrants for the Shift-Absolute versus Relative condition. The number of times each critical location was crossed and the rats' average distance from each location during the probe trial were measured. The latter measure was adapted from the goal proximity measure described by Gallagher, Burwell, and Burchinal (1993). The latency to enter and the amount of time spent in a circular region $(66 \mathrm{~cm}$ in diameter) centered around each of the critical locations were also measured.

\section{Results}

All statistical tests reported here are from univariate analyses of variance (ANOVAs) and are significant at $p<.05$ unless otherwise noted.

Hidden platform training. Latency data were averaged for each of the three training trial blocks for each day and analyzed with a repeated-measures ANOVA with trial block (1-9), pool position ( 1 or 2 ), and platform location within the room (A, B, or C) as factors. There was a main effect of trial block, $F(8,224)=$ 87.64 , which resulted from a decrease in latency to navigate to the platform across trial blocks $\left(M_{\text {Block 1 }}=27.31 \mathrm{~s}, S D=11.64\right.$; $\left.M_{\text {Block } 9}=3.39 \mathrm{~s}, S D=1.29\right)$. There were, however, no main effects of pool position or platform location (both $p s>.16$ ), and none of the two-way interactions involving trial block reached statistical significance (all $p s>.21$ ). The Pool Position $\times$ Platform Location interaction and the three-way interaction were not computed because pool position and platform location could not be completely crossed. To determine whether there were differences in learning based on the particular combinations of pool position and platform location, we conducted an ANOVA with trial block and combination of pool position and platform location as a between-subjects factor with four levels. There was no detectable effect of the combination of pool position and platform location and this factor did not interact with trial block (all $p \mathrm{~s}>.30$ ).

No-platform probe trial. Representative probe trial swim paths for rats from each condition of Experiment 1 are shown in Figure 2, and condition means for each of the probe trial dependent measures are shown in Figure 3. All 16 rats in the No Shift condition navigated to the absolute spatial location of the platform during training first. Comparisons of each dependent measure for the two critical locations revealed that rats in the No Shift condition navigated faster to the absolute region than to the opposite region, $F(1,15)=86.85$; spent more time in the absolute region, $F(1,15)=88.67$; crossed the absolute location more frequently, $F(1,15)=48.13$; and navigated closer to the absolute location, $F(1,15)=87.47$.

All 8 rats in the Shift-Absolute versus Relative condition navigated to the relative region first rather than navigating to the absolute spatial location within the room. Supplementary Video 1 shows a representative trial for a single rat in the Shift-Absolute versus Relative condition. Rats in this condition navigated faster to the relative region than to the absolute region, $F(1,7)=28.27$; spent more time in the relative region, $F(1,7)=27.35$; crossed the relative location more frequently, $F(1,7)=10.80$; and navigated closer to the relative location, $F(1,7)=18.33$.

All 8 rats in the Shift-Relative Only condition navigated to the relative region of the pool first rather than navigating to the region opposite the relative location. Rats in this condition navigated faster to the relative region than to the opposite region, $F(1,7)=26.12$; spent more time in the relative region, $F(1,7)=8.75$; crossed the relative 


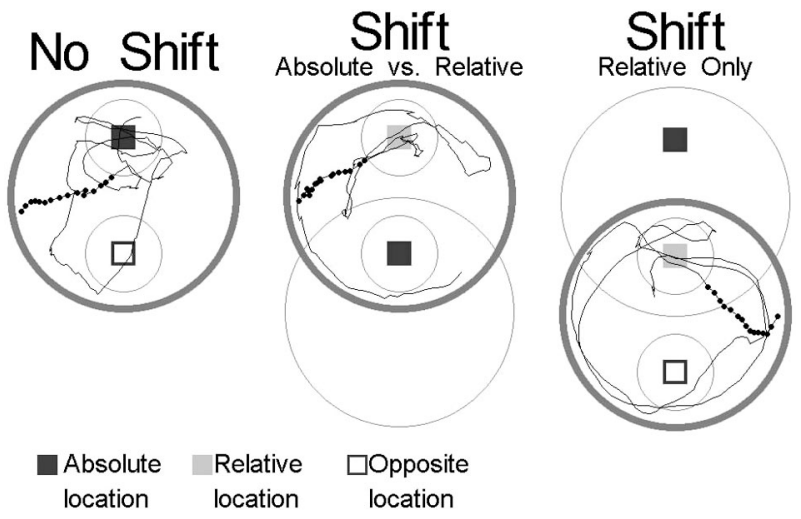

Figure 2. Representative swim paths during the no-platform probe trial for each condition of Experiment 1. Paths were selected for rats with median latencies to enter the preferred region during the probe trial (absolute for the No Shift condition and relative for the shift conditions). The large, thin circles shown for the shift conditions indicate the pool position used during hidden platform training. The thick circles indicate the pool position during the probe trial. The thin circles within the pool mark the two critical regions $(66 \mathrm{~cm}$ diameter) around the absolute (dark gray square), relative (light gray square), or opposite location (open square) used for analysis. The initial trajectory, defined as the path taken from the release point until one of the two critical circular regions was entered, is shown in filled black circles. The remainder of the path for the probe trial is shown as a thin black line.

location more frequently, $F(1,7)=6.83$; and, on average, navigated closer to the relative location, $F(1,7)=15.17$.

Because the design of Experiment 1 does not allow for an ANOVA with condition and location as factors (condition and critical location are not fully crossed), direct comparisons of several condition means were conducted to evaluate the effects of shifting the pool within the room reference frame. The first set of comparisons was conducted for the absolute location measures in the No Shift and Shift-Absolute versus Relative conditions. Rats in the No Shift condition navigated faster to the absolute region than did rats in the Shift-Absolute versus Relative condition, $F(1,22)=64.19$; spent more time in the absolute region, $F(1,22)=65.85$; crossed the absolute location more frequently, $F(1,22)=31.03$; and navigated closer to the absolute location, $F(1,22)=62.84$.

Comparisons for the relative location in the Shift-Absolute versus Relative condition with the opposite location for the No Shift condition revealed significant differences for all dependent measures: latency to navigate to the region of interest (No Shift $>$ Shift), $F(1,22)=35.30$; time in region (No Shift $<$ Shift), $F(1$ $22)=63.45 ;$ number of location crossings (No Shift $<$ Shift), $F(1$, $22)=14.55$; and average proximity to the region of interest (No Shift $>$ Shift), $F(1,22)=68.56$.

Comparisons for the relative location in the Shift-Absolute versus Relative condition and the absolute location for the No Shift condition were also conducted. Rats in the No Shift condition navigated faster to the absolute region than rats in the ShiftAbsolute versus Relative condition navigated to the relative region, $F(1,22)=4.65$; spent more time in the absolute region than Shift-Absolute versus Relative spent in the relative region, $F(1$, $22)=7.22$; and crossed the absolute location more frequently than Shift-Absolute versus Relative rats crossed the relative location, $F(1,22)=7.49$. The condition differences for average proximity to location failed to reach significance, $F(1,22)=3.74, p=.067$, although the No Shift rats navigated more closely to the absolute
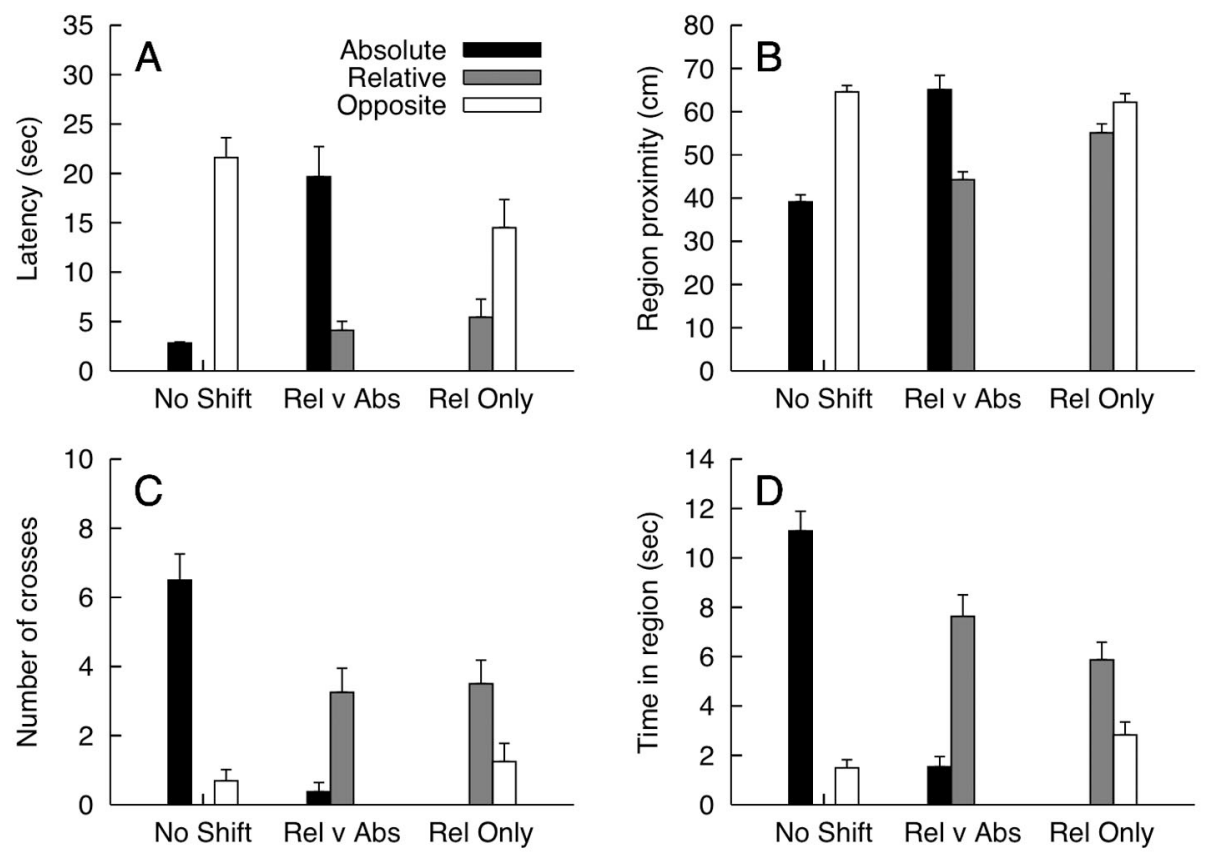

Figure 3. Mean (+ SEM) probe trial dependent measures for each condition of Experiment 1. "Rel v Abs" and "Rel Only" refer to the Shift-Absolute vs. Relative and Shift-Relative Only conditions, respectively. A: Latency to enter the $66 \mathrm{~cm}$ diameter circular region around the two locations of interest. B: Mean distance (proximity) from the two critical locations. C: Number of times each critical location was crossed. D: Time spent in each of the two critical circular regions. 
location than the Shift-Absolute versus Relative rats navigated to the relative location.

Finally, measures for the absolute location in the Shift-Absolute versus Relative condition and the opposite location for the No Shift and Shift-Relative Only condition were subjected to a oneway ANOVA with condition as a single factor. None of the effects reached statistical significance: latency $(p=.10)$, time in region ( $p=.07)$, platform location crosses $(p=.37)$, and region proximity $(p=.65)$.

\section{Discussion}

Rats were trained to navigate to a fixed hidden platform, after which the pool was repositioned in the room such that animals could navigate either to the absolute spatial location within the room or to the relative location within the pool. This manipulation resulted in a clear pattern of data. When the pool was repositioned, rats navigated to the relative location first, crossed the relative location more frequently, spent more time in the relative region, and navigated more closely to the relative location. Further, rats in the Shift-Absolute versus Relative condition had latencies and average proximity scores for the relative location that were comparable with those of rats in the No Shift condition for the trained (absolute) location. This set of observations is particularly impressive because the Shift rats were never explicitly trained to navigate to the relative location. The present results would be expected if animals navigate in a particular direction that has been associated with finding the platform (within the room and apparatus) and search at the appropriate distance from the pool wall. Given that the rats in the Shift-Absolute versus Relative condition treated the relative location in much the same way that the No Shift rats treated the absolute location, directional responding could account for performance in the standard Morris water task where the pool remains in the same position. The results are inconsistent with the hypothesis that rats learn to swim to an absolute spatial location on the basis of distal cues alone. Of course, in addition to learning a directional response during training, rats may also have learned the absolute location of the platform and our results may simply reflect a preference for directional responding over navigation to the absolute location when the pool is repositioned.

The preference for the relative location in Shift animals must reflect control by cues other than those from the pool wall alone, as the pool wall does not disambiguate the relative and absolute locations. The most obvious source of such control is the distal visual cues. Further, because the precise location where the search behavior occurred was never explicitly trained, the observations that Shift-Absolute versus Relative rats cross over the relative location more frequently than they do the absolute location and search at the appropriate distance from the pool wall at the relative location are difficult to explain in terms of control by distal cues alone. The observed search behavior must also be controlled by another source, and the apparatus wall is the only feature in the environment that could effectively control focused search at the relative location. The collective results of the probe trial, thus, indicate that navigation to and searching at a putative goal location when the pool is shifted are controlled by both the distal cues and the pool wall. Experiment 2 was undertaken to determine whether, during a single trial, these sources of control combine with a third salient source of control, a single visual cue that marks the platform location, to control directional responding or navigation to absolute locations.

\section{Experiment 2}

The processes involved in place navigation to a hidden platform in the Morris water task are often contrasted with cued navigation, in which a single visual cue marks the platform location. Thus, the rat need only learn to swim to this cue and there is no requirement that it learn to navigate on the basis of distal visual cues or apparatus. In some cases, a cue that is co-localized with the platform overshadows the distal cues (Redhead, Roberts, Good, \& Pearce, 1997), whereas other studies have shown that animals can navigate to the platform location on the basis of distal cues when a visible platform is removed (Whishaw, Mittelman, Bunch, \& Dunnett, 1987). Hamilton, Rosenfelt, and Whishaw (2004) demonstrated that some aspects of navigation, such as the direction of the initial trajectory to a visible platform, are controlled by distal cues even when the visible platform overshadows the distal cues with respect to other behaviors (e.g., persistence in searching).

If distal cues provide a source of control during cued navigation, then relocating the pool after training should yield evidence regarding the type of control provided by the distal room cues during cued navigation. In Experiment 2, rats were trained to swim to a platform marked by a conspicuous visual cue and two critical test trials were conducted. For the first test, the pool was shifted by 75 $\mathrm{cm}$ (e.g., from Location 1 to Location 2) and the visible platform was placed in either the absolute (trained) spatial location or in the relative location within the pool. If distal cues control directional responding even with a cued platform, then performance should be disrupted by shifting the pool and keeping the cued platform in the absolute spatial location but should be unaffected if the platform is kept in the relative spatial location in the apparatus. For the second test trial, the pool was either shifted or remained in the trained location and the platform and cue were removed from the pool for a probe trial. If the presence of the cued platform during training does not alter the way in which the distal cues and apparatus wall control the trajectory and persistence in searching, then rats should navigate to and persist in searching at the relative location as observed in Experiment 1. It is, however, also possible that the presence of the cue during training may support navigation to the absolute spatial location rather than directional responding when the pool is repositioned.

\section{Method}

Subjects. Subjects were 15 male Long-Evans rats (Rattus norvegicus; Charles River Laboratories, Wilmington, MA) that were approximately 90 days of age at the beginning of Experiment 2. All animals were housed with one cage mate in plastic cages on a 12-hr light-dark cycle with food and water available ad libitum. Behavioral testing was performed during the light phase from approximately $1200-1300$.

Apparatus. The room and pool were the same as that used in Experiment 1. A black plastic ball $(10 \mathrm{~cm}$ diameter) attached to a metal rod served as a proximal cue co-localized with the platform. The metal rod was placed in the center of the platform such that the bottom of the plastic ball was $10 \mathrm{~cm}$ above the platform surface.

Design and procedure. In Experiment 2, all rats were given 12 cued platform training trials during each of three daily sessions for a total of 36 trials. Two pool locations were used and the platform was always placed at Location B (see Figure 1). Eight rats were trained with the pool in Position 1, and 7 rats were trained with the 
pool in Position 2 (see Table 2). During each daily session of 12 trials, rats completed three blocks of four trials during which each of four possible release points around the perimeter of the pool (see Figure 1) was used once. The order of release points followed a pseudorandom sequence. Rats were released facing the wall of the pool and were allowed to remain on the platform for $5 \mathrm{~s}$ before being returned to their holding cage.

Two test trials were conducted. The pool positions and platform locations used during training and testing are listed in Table 2. The first test trial immediately followed the fourth training trial on Day 3 . The pool was shifted $75 \mathrm{~cm}$ from the trained position for all rats. For the 8 rats assigned to the Absolute condition, the cued platform was positioned at the absolute location used during training (Location B). For the 7 rats assigned to the Relative condition, the cued platform was moved along with the pool such that it remained in the same relative location within the pool. Two novel release points (north or south) were used for the first test trial, and the trial ended when the rat found the platform. Latency and path length to navigate to the escape platform were measured.

Following the first test trial, the remaining 8 training trials were conducted with the cued platform and pool in the original, trained locations. On completion of the final training trial on Day 3, a no-platform probe trial was conducted. For the 7 rats assigned to the No Shift condition, the pool remained in the trained location. A roughly equal number of rats from the Absolute and Relative test trial conditions were assigned to the No Shift condition (see Table 2). For the 8 rats assigned to the Shift condition, the pool was shifted by $75 \mathrm{~cm}$ (from Position 1 to 2 or from Position 2 to 1). An equal number of rats $(n=4)$ from the absolute and relative conditions from the first test trial were assigned to the shift condition. The probe trial lasted for $30 \mathrm{~s}$, and the north or south release points were used. The dependent measures were the same as those for the probe trial of Experiment 1. The particular sequence of test and training trials on Day 3 was selected so that all rats received four training trials prior to the first test trial, followed by eight additional training trials prior to the no-platform probe trial. As the no-platform probe trial was an extinction trial, the pool relocation test trial with the cued platform was conducted first because we reasoned that it would be less likely to affect performance on the subsequent training trials and the no-platform probe trial.

Table 2

Pool Positions and Platform Locations (See Figure 1) Used During Cued Platform Training, the Cued Platform Test Trial, and the No-Platform Probe Trial of Experiment 2

\begin{tabular}{|c|c|c|c|c|}
\hline \multirow{2}{*}{$\begin{array}{c}\text { Training trial } \\
\text { (position: platform) }\end{array}$} & \multicolumn{2}{|c|}{ Cued platform test } & \multicolumn{2}{|c|}{ No-platform probe } \\
\hline & Position: platform & Condition & Position & Condition \\
\hline 1: B & 2: B & Absolute & 1 & No Shift \\
\hline 1: B & 2: B & Absolute & 2 & Shift \\
\hline $2: B$ & 1: B & Absolute & 1 & Shift \\
\hline 2: B & $1: \mathrm{B}$ & Absolute & 2 & No Shift \\
\hline 1: B & 2: $\mathrm{C}$ & Relative & 1 & No Shift \\
\hline 1: B & 2: $\mathrm{C}$ & Relative & 2 & Shift \\
\hline $2: B$ & 1: A & Relative & 1 & Shift \\
\hline $2: \mathrm{B}$ & 1: A & Relative & 2 & No Shift \\
\hline
\end{tabular}

Note. Each row represents a sequence of training and testing used for two rats, with the exception of the final sequence, which was used for one rat.

\section{Results}

Cued platform training. Latency data were averaged for each of the three training trial blocks (four trials each) conducted on each day and analyzed with repeated-measures ANOVA with trial block (1-9) and pool position (1 or 2) as factors. There was a main effect of trial block, $F(8,104)=126.28$, which resulted from a decrease in escape latency across trial blocks $\left(M_{\text {Block } 1}=19.08 \mathrm{~s}\right.$, $\left.S D=5.52 ; M_{\text {Block } 9}=2.70 \mathrm{~s}, S D=0.35\right)$. The main effect of pool position $(p=.71)$ and the Pool Position $\times$ Trial Block interaction $(p=.08)$ were not significant.

Absolute versus relative test trial. Representative test trial swim paths for rats from the Absolute and Relative conditions are shown in Figure 4, and condition means for latency and path length to navigate to the escape platform are shown in Figure 5. Test trials for all rats are shown in Supplementary Video 2. In the Absolute condition, 5 of the 8 rats showed significant disruptions in performance even though the cue above the platform was visible just as it was during training. Although 3 rats (Numbers 37, 38, and 41 in Supplementary Video 2) navigated more or less directly to the escape platform in the absolute location, Rats 37 and 41 clearly hesitated during their swim, resulting in longer escape latencies. In the Relative condition, 6 of the 7 rats navigated directly to the cued platform in the relative location. One rat (Rat 45 in Supplementary Video 2) began a trajectory in the general direction of the absolute location but then quickly altered its trajectory and navigated to the cued platform in the relative location. These qualitative differences in paths between the two conditions were accompanied by significant condition differences (Relative $<$ Absolute) in latency to navigate to the platform, $F(1,13)=9.28$, and path length to navigate to the platform, $F(1,13)=7.27$.

No-platform probe trial. Representative probe trial swim paths for rats from the No Shift and Shift conditions of Experiment 2 are shown in Figure 6, and condition means for each of the dependent measures are shown in Figure 7.

In the No Shift condition, 6 of the 7 rats navigated directly to the absolute region of the pool where the platform was located during training. One rat navigated first to the opposite quadrant of the pool. Rats in the No Shift condition navigated faster to the absolute region than to the opposite region, $F(1,6)=13.49$; spent more time in the absolute region, $F(1,6)=11.42$; and, on average, navigated closer to the absolute location, $F(1,6)=5.90$. Although rats in the No Shift condition crossed the absolute location more than twice as frequently as they crossed the opposite location, this difference was not significant ( $p=.34$ ).

In the Shift condition, 7 of the 8 rats navigated directly to the relative region of the pool where the platform was located during training. One rat navigated to the absolute region first. Rats in the Shift condition navigated faster to the relative region than to the absolute region, $F(1,7)=7.54$, and, on average, navigated closer to the relative location, $F(1,7)=5.47$. Although rats in the Shift condition crossed the relative location more than they crossed the absolute location, this difference was not significant, and the condition difference for time in region also failed to reach significance (both $p \mathrm{~s}>.23$ ).

To evaluate the effects of shifting the pool on performance, we conducted an ANOVA with condition (No Shift vs. Shift) and location (absolute vs. other [relative or opposite quadrant]) as factors. There were no significant main effects of condition or location for any of the dependent measures (all $p \mathrm{~s}>$.07). The 


\section{Absolute Relative}

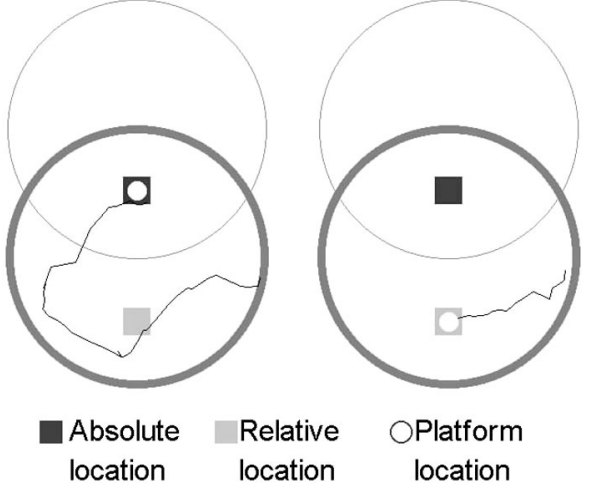

Figure 4. Representative swim paths for the absolute and relative conditions during the cued platform test trial of Experiment 2. Paths were selected for rats with median latencies to navigate to the cued platform. The large, thin circles indicate the pool position used during training, and the thick circles indicate the pool position during the test trial. The dark gray square marks the absolute spatial location (i.e., the trained location) within the distal room reference frame. The light gray square marks the relative location of the platform within the pool. The white circle marks the location of the platform during the test trial.

Condition $\times$ Location interaction for number of platform crosses did not reach significance $(p=.20)$; however, significant interactions for latency, $F(1,13)=21.94$; time in region, $F(1,13)=$ 12.49; and region proximity, $F(1,13)=10.04$, were observed. As a result, we present the results of pairwise comparisons among condition means for these measures.

To simplify the presentation of these comparisons, we compared condition means separately for the absolute and opposite quadrant locations. Consistent with the observations noted above, rats in the No Shift condition navigated to the absolute region faster, $F(1$, $13)=7.30$; spent more time in the absolute region, $F(1,13)=$ 10.34; and navigated closer, on average, to the absolute location, $F(1,13)=9.89$, than did rats in the Shift condition.

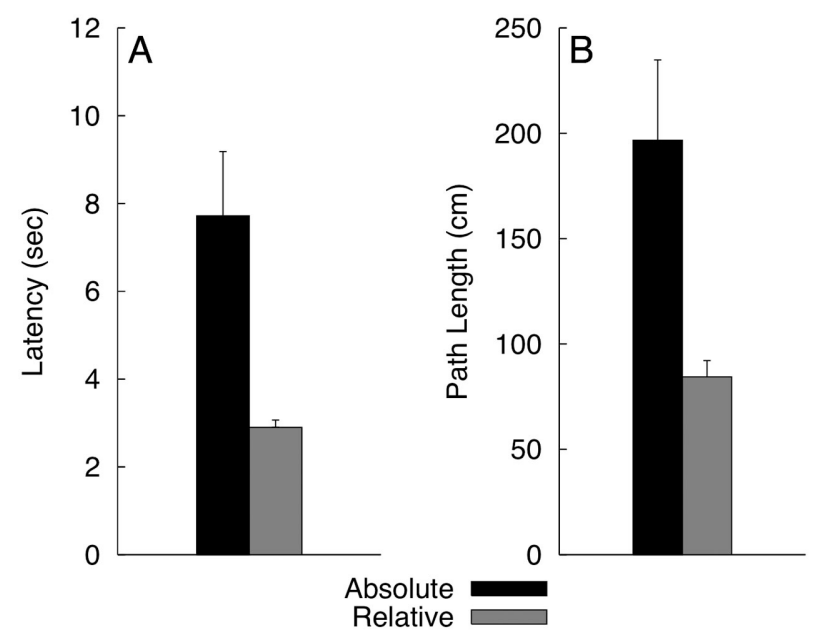

Figure 5. Mean (+ SEM) latency (A) and path length (B) for rats in the absolute and relative conditions to navigate to the escape platform during the cued platform test trial from Experiment 2.
A similar pattern of results was obtained in comparisons of the opposite location measures (No Shift condition) and relative location measures (Shift condition). Rats in the Shift condition navigated to the relative region faster than No Shift rats reached the region opposite the absolute location, $F(1,13)=15.79$. Shift rats also spent more time in the relative region, $F(1,13)=8.23$. Animals in the Shift condition also navigated closer to the relative location than rats in the No Shift condition navigated to the opposite quadrant location; however, this difference failed to reach significance $(p=.09)$.

Of particular importance are comparisons of measures related to the preferred region for each condition. Comparisons of the absolute location measures for the No Shift condition and the relative location measures for the Shift condition failed to detect significant differences for latency and average distance from the location of interest (both $p \mathrm{~s}>.22$ ). Rats in the No Shift condition, however, did spend more time in the absolute region than Shift animals spent in the relative region, $F(1,13)=6.28$.

The question of whether the rats in each condition navigated to and searched at the nonpreferred region differently were addressed by direct comparisons of the absolute location measures for the Shift condition and the opposite location measures for the No Shift condition. These comparisons failed to detect significant differences for latency, average distance from the location of interest, and time spent in the region of interest (all $p \mathrm{~s}>.08$ ).

\section{Discussion}

The results of Experiment 2 clearly indicate that navigation to a cued platform involves multiple sources of control, including the distal visual cues and the pool wall. Further, consistent with the

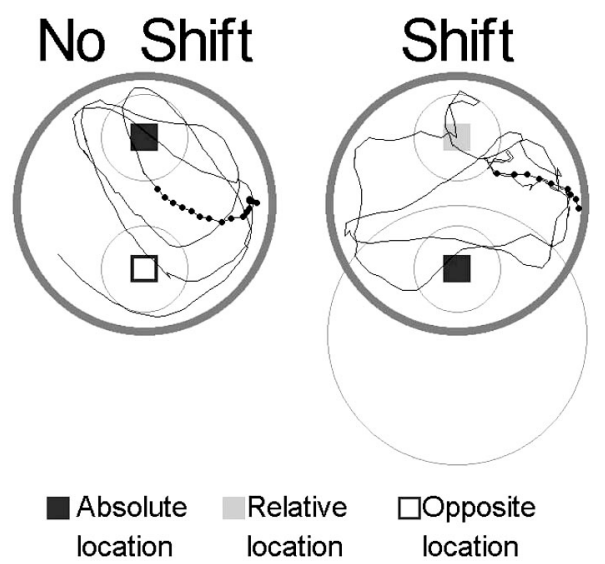

Figure 6. Representative swim paths for the Shift and No Shift conditions during the no-platform probe trial of Experiment 2. Paths were selected for rats with median latencies to enter the preferred region during the probe trial (absolute for the No Shift condition and relative for the Shift condition). The large, thin circles shown for the Shift condition indicate the pool position used during training. The thick circles indicate the pool position during the probe trial. The thin circles within the pool mark the two critical regions (66 $\mathrm{cm}$ diameter) around the absolute (dark gray square), relative (light gray square), or opposite location (open square) used for analysis. The initial trajectory, defined as the path taken from the release point until one of the two critical circular regions was entered, is shown in filled black circles. The remainder of the path for the probe trial is shown as a thin black line. 

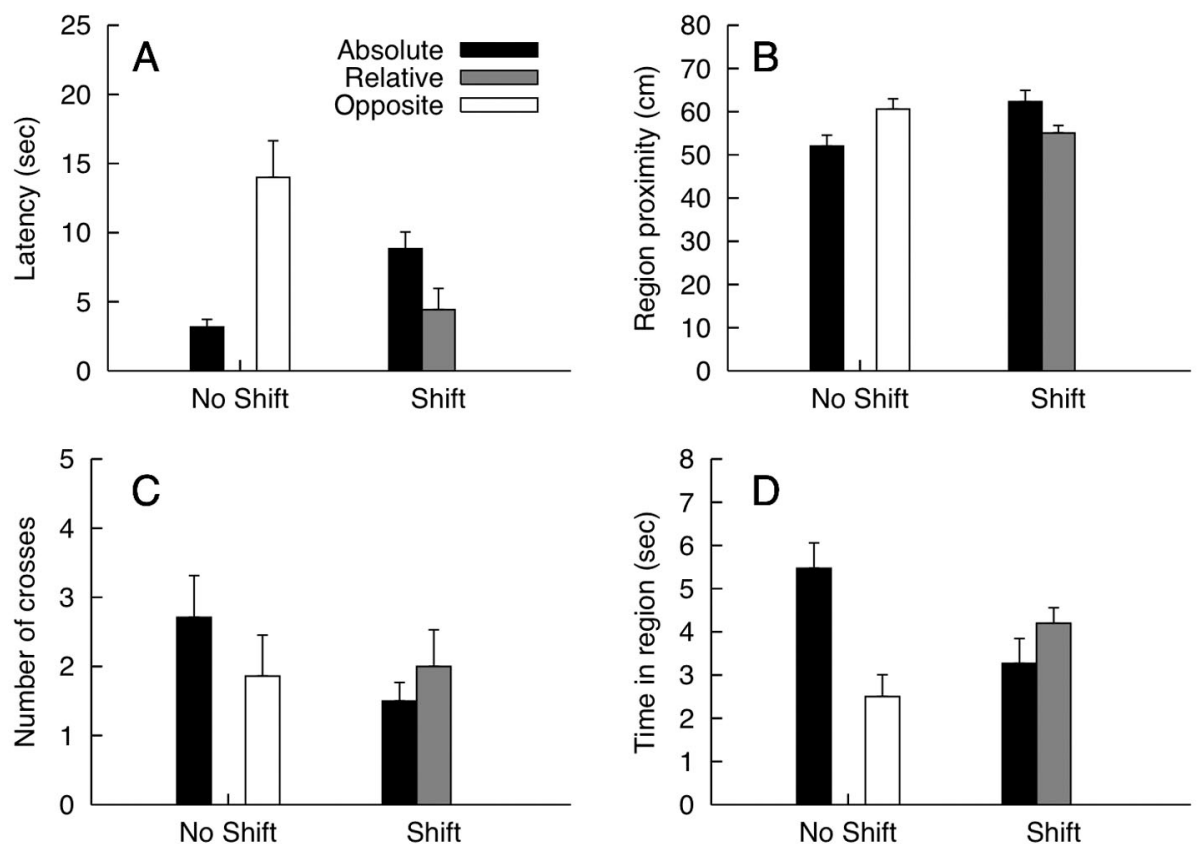

Figure 7. Mean $(+S E M)$ probe trial dependent measures for the No Shift and Shift conditions of Experiment 2. A: Latency to enter the $66 \mathrm{~cm}$ diameter circular region around the two locations of interest. B: Mean distance (proximity) from the two critical locations. C: Number of times each critical location was crossed. D: Time spent in each of the two critical circular regions.

findings from Experiment 1, the distal cues controlled directional responding rather than navigation to the absolute spatial location of the platform. Perhaps the most striking observation from Experiment 2 is the large disruption in cued navigation when the pool was shifted and the platform was kept in the same absolute location in a familiar room (see Supplementary Video 2). The degree of disruption observed here is comparable with that observed by Hamilton et al. (2004) when rats navigated to a familiar visible platform in a completely novel distal cue environment. The fact that shifting the pool within the room did not disrupt cued navigation when the platform was kept in the relative spatial location indicates that the distal cues control directional responding and that the disruptions observed in the Absolute condition were not simply due to a more generalized disruption caused by changing the relationship between the pool and the distal cues.

The results of the no-platform probe trial correspond well with the results of the first test trial. Shift rats navigated to the relative location first, navigated closer to the relative location than to the absolute location, and showed evidence of persisting at the relative location more than at the absolute location. This pattern supports the idea that the distal cues, pool wall, and platform cue all control behavior during a single trial and that the presence of a cue co-localized with the platform does not fundamentally alter how distal cues and the pool wall control navigation. Considered together, the results of Experiments 1 and 2 provide strong support for navigation on the basis of direction and distance information provided by the room and apparatus cues, respectively.

\section{Experiment 3}

Given the clear pattern of data in support of directional responding from Experiments 1 and 2, we examined two additional aspects of the procedures that may have contributed to the observed results. First, performance was clearly at asymptote after 8-12 training trials; however, a total of 36 training trials were given. In dry land mazes, it is claimed that rats navigate to places early during training but show a shift to response-based performance later during training (Chang \& Gold, 2003; Packard \& McGaugh, 1996). If this behavioral pattern holds generality, then it is possible that the rats tested in Experiments 1 and 2 navigated to the absolute place where the platform was located during the initial stages of training but shifted to a directional response strategy later in training. The methods used in Experiments 1 and 2 should effectively rule out other forms of responding, such as taking specific routes to the platform, because the pool was repositioned in the room and a novel release point was used for the critical probe trial. It is, however, conceivable that movement in a particular direction in the room and apparatus becomes the predominant behavior only after overtraining. A second methodological feature of the previous experiments that may have supported directional responding over place navigation is the brief duration ( $5 \mathrm{~s}$ ) the rats were allowed to stay on the platform. Several studies have presented positive evidence that rats can learn to place navigate on the basis of experience viewing the distal cues from the platform location (Devan et al., 2002; Keith \& McVety, 1988; Pearce, Roberts, Redhead, \& Prados, 2000; Sutherland \& Linggard, 1982; but see Sutherland et al., 1987, and Chew, Sutherland, \& Whishaw, 1989), thus, limited platform experience could have had a detrimental effect on learning to navigate to the place where the platform was located.

Either or both of these procedural elements could have resulted in a preference for directional responding over navigating to the platform location. To examine this possibility, we gave rats a total 
of eight hidden platform training trials and allowed them to stay on the platform for $30 \mathrm{~s}$ at the end of each trial. The pool was then repositioned as in Experiments 1 and 2 and navigation to the relative and absolute locations was assessed. If overtraining and limited experience from the platform were responsible for the directional responding observed in Experiments 1 and 2, then the results of Experiment 3 should reveal no preference for the relative location. Further, if animals shift from navigating to places early in training to directional responding later in training, then rats should navigate to the absolute location when the pool is repositioned.

\section{Method}

Subjects. Subjects were 16 experimentally naive male LongEvans rats (Rattus norvegicus; Charles River Laboratories, Wilmington, MA) that were approximately 90 days of age at the beginning of the experiment. All animals were pair housed in plastic cages on a 12-hr light-dark cycle with food and water available ad libitum. Behavioral testing was performed during the light phase from approximately 1200-1300.

Apparatus. The room and pool were the same as those used in Experiments 1 and 2.

Design and procedure. Experiment 3 consisted of a training phase followed by a single no-platform probe trial. During training, all rats were given eight hidden platform trials during a single session. The animals were released twice from each of the four release points around the perimeter of the pool (see Figure 1) in a pseudorandom sequence. Rats were retrieved by the experimenter and placed on the platform if the trial duration exceeded $60 \mathrm{~s}$. Regardless of whether the rats were placed on the platform or swam to the platform, they were allowed to remain on the platform for $30 \mathrm{~s}$ before being returned to the holding cage. Two pool positions (1 and 2) were used, and the platform was always at Location B in the room (see Figure 1). An equal number of rats $(n=8)$ were randomly assigned to each of the two possible combinations of pool position and platform location. The latency to navigate to the platform was measured for each trial.

On completion of the final training trial, a single no-platform probe trial was conducted. The probe trial lasted for $30 \mathrm{~s}$, and animals were released from one of two novel release points (north or south; see Figure 1). Half of the animals from each combination of pool position and platform location were assigned to either a Shift condition $(n=8)$ in which the pool was moved $75 \mathrm{~cm}$ from the trained position to the other possible pool position or to a No Shift condition $(n=8)$ in which the pool remained in the same position used during training. The Shift condition of Experiment 3 corresponds to the Shift-Absolute versus Relative condition from Experiment 1 (see Table 1), and the No Shift condition was the same as that of Experiment 1. Four dependent measures were taken for the probe trial, as in Experiments 1 and 2.

\section{Results}

Hidden platform training. Escape latencies for each of the eight training trials were analyzed with repeated-measures ANOVA with trial (1-8) and pool position (1 or 2) as factors. There was a main effect of trial, $F(7,98)=28.52$, which resulted from a decrease in escape latency across trials $\left(M_{\text {Trial } 1}=51.25 \mathrm{~s}\right.$, $\left.S D=11.11 ; M_{\text {Trial } 8}=4.81 \mathrm{~s}, S D=2.69\right)$. The main effect of pool position was not significant, and there was no significant Pool Position $\times$ Trial interaction (both $p \mathrm{~s}>.08$ ).
A separate analysis was conducted with trial and probe trial condition (No Shift or Shift) as factors. The condition main effect was not significant nor was there a significant Condition $\times$ Trial interaction (both $p \mathrm{~s}>$.16). The asymptotic latency for hidden platform training during Experiment 1 was $4.85 \mathrm{~s}$ (averaged over the last six blocks). This level of performance was not achieved until Trial 8 in Experiment 3, and means for all previous trials were $7.5 \mathrm{~s}$ or greater. A comparison of condition means for Trial 8 was not significant $\left(p=.17 ; M_{\text {no shift }}=3.88 \mathrm{~s}\right.$ vs. $M_{\text {shift }}=5.75 \mathrm{~s}$ ).

No-platform probe trial. Representative probe trial swim paths for rats from the No Shift and Shift conditions of Experiment 3 are shown in Figure 8, and condition means for each of the dependent measures are shown in Figure 9.

In the No Shift condition, all 8 rats first navigated to the absolute region where the platform was located during training prior to navigating to the opposite region, although the trajectories to the absolute location were less direct than those observed with more extensive training in Experiment 1. Rats in the No Shift condition navigated faster to the absolute region than to the opposite region, $F(1,7)=46.11$; spent more time in the absolute region, $F(1,7)=$ 42.88; navigated closer to the absolute location, $F(1,7)=20.86$; and crossed the absolute platform location more frequently than the opposite location, $F(1,7)=20.24$.

In the Shift condition, 6 of the 8 rats first navigated directly to the relative region where the platform was located during training prior to navigating to the absolute region. One rat entered the absolute region first, but only by a few centimeters, and then showed a clear preference for the relative location during the remainder of the probe trial. Another rat left the release point slowly and followed the pool wall into the absolute region but did not show a clear preference for either location during the probe

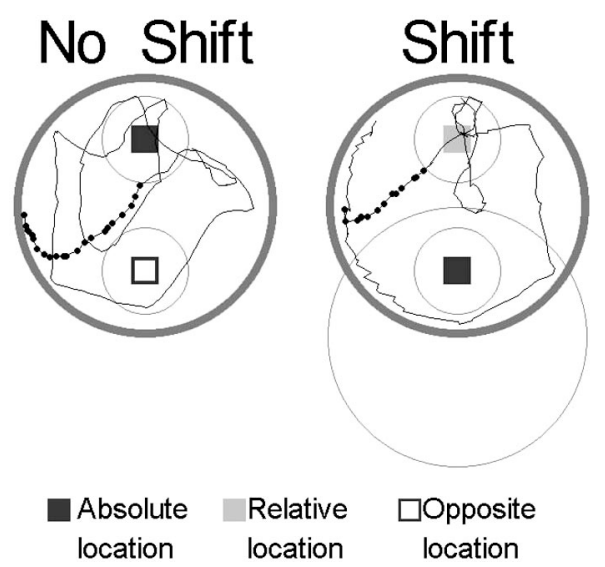

Figure 8. Representative swim paths for the shift and No Shift conditions during the no-platform probe trial of Experiment 3. Paths were selected for rats with median latencies to enter the preferred region during the probe trial (absolute for the No Shift condition and relative for the shift condition). The large, thin circles shown for the shift condition indicate the pool position used during training. The thick circles indicate the pool position during the probe trial. The thin circles within the pool mark the two critical regions (66 cm diameter) around the absolute (dark gray square), relative (light gray square), or opposite location (open square) used for analysis. The initial trajectory, defined as the path taken from the release point until one of the two critical circular regions was entered, is shown in filled black circles. The remainder of the path for the probe trial is shown as a thin black line. 

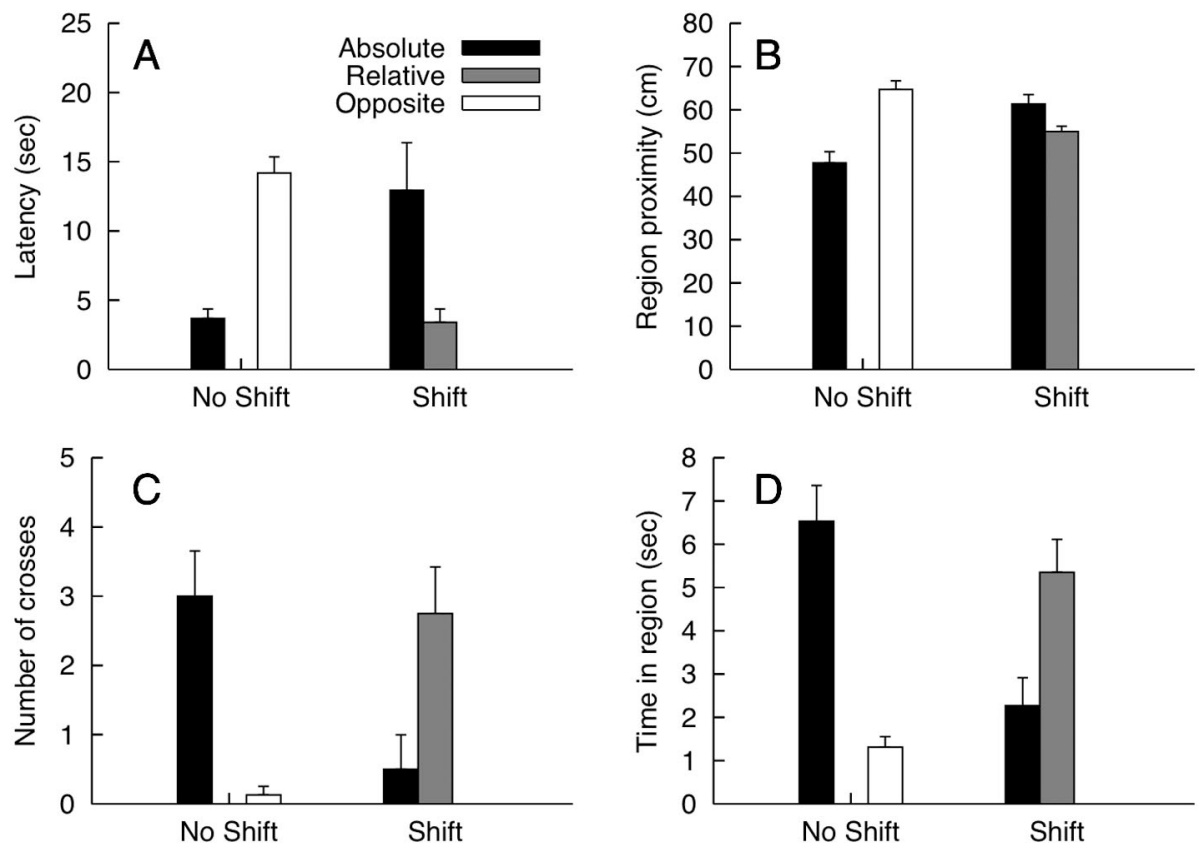

Figure 9. Mean $(+S E M)$ probe trial dependent measures for the No Shift and Shift conditions of Experiment 3. A: Latency to enter the $66 \mathrm{~cm}$ diameter circular region around the two locations of interest. B: Mean distance from the two critical locations. C: Number of times each critical location was crossed. D: Time spent in each of the two critical circular regions.

trial. Rats in the Shift condition navigated faster to the relative region than to the absolute region, $F(1,7)=5.73$; spent more time in the relative region, $F(1,7)=5.85$; navigated closer to the relative location, $F(1,7)=5.65$; and crossed the relative platform location more frequently than the absolute location, $F(1,7)=$ $5.30, p=.055$.

To evaluate the effects of shifting the pool on performance, we conducted an ANOVA with condition (No Shift vs. Shift) and location (absolute vs. other [relative or opposite quadrant]) as factors. There were no significant main effects of condition for any dependent measure (all $p \mathrm{~s}>.28$ ). There was a significant main effect of location for average proximity (absolute $<$ other), $F(1$, $14)=5.37$, indicating that, overall, rats navigated closer to the absolute region. The remaining main effects of location failed to reach significance (all $p \mathrm{~s}>.17$ ). There were significant Location $\times$ Condition interactions for latency, $F(1,14)=21.97$; time in region, $F(1,14)=30.58$; platform crosses, $F(1,14)=19.78$; and region proximity, $F(1,14)=25.96$. As a result, we present the results of pairwise comparisons among condition means for these measures.

To simplify the presentation of these comparisons, we compared condition means separately for the absolute and opposite quadrant locations. Consistent with the observations noted above, rats in the No Shift condition navigated faster to the absolute region, $F(1$, $14)=5.16$; spent more time in the absolute region, $F(1,14)=$ 16.43; navigated closer, on average, to the absolute location, $F(1$, $14)=15.45$; and crossed the absolute platform location more frequently, $F(1,14)=9.21$, than did rats in the Shift condition. Rats in the Shift condition navigated faster to the relative region than No Shift rats navigated to the opposite region, $F(1,14)=$ 49.84; spent more time in the relative region than No Shift rats spent in the opposite region, $F(1,14)=25.37$; navigated closer to the relative location than No Shift navigated to the opposite location, $F(1,14)=17.27$; and crossed the relative location more frequently than No Shift rats crossed the opposite location, $F(1$, $14)=11.23$.

Direct comparisons of the absolute location measures for the No Shift condition and the relative location measures for the Shift condition were undertaken to determine whether there were detectable differences in how animals from each condition navigated to and searched at the preferred location. These comparisons failed to detect significant condition differences for latency, time in the region of interest, and number of platform crosses $(p s>.31)$. Rats in the No Shift condition, however, navigated more closely to the absolute location than Shift rats navigated to the relative location, $F(1,14)=6.35$.

The question of whether the rats in each condition differentially navigated to and searched at the nonpreferred region were addressed by direct comparisons of the absolute location measures for the Shift condition and the opposite location measures for the No Shift condition. These comparisons failed to detect significant differences for any of the dependent measures (all $p \mathrm{~s}>.18$ ).

\section{Discussion}

If animals navigate to absolute spatial locations early during training and extended platform placement supports learning to navigate to the precise spatial location of the platform, then giving longer platform experience and fewer training trials should result in a preference for the absolute location. The procedures used in Experiment 3, however, did not alter the pattern of results observed in Experiments 1 and 2. Providing animals with $30 \mathrm{~s}$ of 
platform experience on each trial and providing fewer training trials resulted in a virtually identical pattern of results observed with less platform experience and overtraining. For every measure of performance during the probe trial, rats in the Shift condition showed a clear preference for the relative location when the pool was shifted. As in the previous experiments reported here, navigation to the relative location in the Shift condition was highly similar to navigation to the absolute location in the No Shift condition. Moreover, the Shift rats treated the absolute region in much the same way that rats in the No Shift condition treated an arbitrarily selected region in the opposite side of the pool. Thus, together with the results of Experiments 1 and 2, the outcome of Experiment 3 provides a clear demonstration that rats perform directional responding over navigation to the absolute location in the water task. Of importance, the rats used in Experiment 3 were task naive whereas the rats in Experiment 1 had prior experience in the water task approximately 70 days earlier on Postnatal Days 17-24. Thus, the results of Experiment 3 support the conclusion that the prior water task experience of rats in Experiment 1 did not contribute to the observed directional responding.

The goal in Experiment 3 was to cease training when rats were just reaching asymptotic levels of performance. It could, however, be argued that the rats were already at or approaching asymptotic levels of performance prior to Trial 8. If so, then it would still be possible that the animals transiently used a strategy that would result in navigation to the absolute location (e.g., for a couple of trials) and had already shifted to directional responding by the time the probe trial was conducted. There are good reasons to discount this argument. In observing the behavior of all the animals, it was not until around Trial 7 or 8 that animals clearly took direct paths to the platform; thus, direct navigation to an absolute place or directional responding to the relative location could not have occurred until Trial 7 at the earliest. Indeed, neither group reached asymptotic levels of performance comparable with the values observed in Experiment 1 until Trial 8. Because rats only swam directly to the platform for one or two trials prior to the probe trial, the results of Experiment 3 score against the idea that a shift in response type with overtraining can explain the results of Experiments 1 and 2.

\section{General Discussion}

The results of the three experiments reported here demonstrate a clear preference for navigation to the relative location rather than the absolute platform location when the pool was repositioned. These observations are consistent with the idea that rats navigate to the platform in the Morris water task by swimming in the direction of the platform in the room but are inconsistent with the notion that accurate performance in this task depends on learning to navigate to the precise spatial location of the platform. In addition to directly navigating to the relative location, rats also persisted in searching at the relative location of the platform, indicating that search behaviors typically observed in water task probe trials are still supported by the available cues when the pool is repositioned. The preference for the relative location was not altered by (a) removing the opportunity to navigate to the absolute platform location (Experiment 1), (b) the presence of a cue that marked the platform (Experiment 2), (c) limiting the number training trials to avoid overtraining (Experiment 3), or (d) increasing the duration of experience on the platform during training (Experiment 3). In addition, the results of all three experiments highlight the fact that navigation in the Morris water task is controlled by multiple sources, including the distal cues, pool wall, and, if present, any cue that marks the platform. To explain these observations in terms of multiple sources of control, we propose that navigation to an escape platform in the Morris water task involves a movement vector that consists of at least two processes related to direction and distance information: (a) execution of a trajectory, the directionality of which is based on distal room cues, and (b) search behavior at the appropriate distance from the pool wall. This type of explanation is in clear contrast to proposals that emphasize the ability to navigate to specific places; however, these contrasting accounts and the processes they postulate need not be mutually exclusive. After summarizing the major findings of the three experiments, we consider the results in relation to several behavioral and physiological phenomena that have been demonstrated in navigation tasks and discuss the implications for explanations of navigation in the water task.

In the experiments reported here, rats navigated directly to and persisted in searching at the relative location when the pool was shifted to a different location in the room. Despite this rather large shift in the pool position $(75 \mathrm{~cm})$, inspection of the representative swim paths (see Figures 2, 6, and 8) and the condition means for the probe trial (see Figures 3, 7, 9) reveals that in many respects the Shift rats treat the relative location similar to how the No Shift rats treat the absolute location, particularly with respect to the initial trajectory and distribution of search during the probe trial. It should be noted, however, that there was some disruption in performance for the Shift conditions during probe trials, which could be attributed to perceptual differences related to moving the pool a full $75 \mathrm{~cm}$, release from a novel location, and/or the possibility that the place and direction information were made incompatible. The pattern of data for the opposite location in the No Shift conditions provides an indicator of how rats treated an arbitrary location that was the same distance from the pool wall as the trained platform location. It is interesting to note that rats in the Shift conditions treated the absolute location as if it were an untrained location, comparable with that of the opposite location for the No Shift conditions. Experiment 2 established that distal cues and the pool wall acquire control over navigation when a conspicuous cue marks the platform location, and this control involves directional responding rather than place navigation. The results of Experiment 3 indicate that directional responding is not limited to situations in which animals are overtrained and given limited experience from the platform, as in Experiments 1 and 2. Because evidence for directional responding was obtained with just a few training trials, the present results do not support the idea that rats shift from a place strategy early in training to a responsebased strategy later in training, as has been claimed to occur in dry land mazes (Chang \& Gold, 2003; Packard \& McGaugh, 1996). Rather, it appears that rats performed directional responding early as well as later in training. We did not evaluate the possibility that rats may shift from a preference for directional responding to place navigation with more extensive training. Further, we also did not evaluate whether platform placement training alone supports directional responding rather than place navigation. If it does, then the range of situations in which a directional response is learned in the water task would appear to be broad enough to support the conclusion that this is the primary way in which animals navigate to a fixed platform in the water task. Future studies are planned to 
evaluate the effects of overtraining and platform placement alone on place navigation and directional responding.

Overall, the results of the present study fit nicely with the results of previous maze-learning studies that have used similar manipulations. Blodgett et al. (1949) trained rats in a T maze in which a food reward could be found in the end of one arm. The T maze was repositioned in the room from trial to trial such that some rats were trained to always navigate to the same place in the room, regardless of the direction of movement in the room, whereas others were trained to always move in a particular direction in the room. Animals in the latter condition made significantly fewer errors than did animals that were trained to navigate to a particular place. More recently, Skinner et al. (2003) reported similar results in a task in which rats were trained to navigate to one corner of a square open field. The open field was repositioned from trial to trial such that the reinforced corner was always in the same spatial location in the room, regardless of the apparatus position, or was always found by moving in a particular direction in the room. Animals in the directional responding condition met criterion significantly faster than did animals trained to navigate to a specific place.

Although the studies by Blodgett et al. (1949) and Skinner et al. (2003) performed the critical manipulations during training rather than during a single test trial, as was done here, the patterns of data are highly consistent with our findings. Our decision to evaluate the effects of shifting the pool during test trials rather than during acquisition was based on the desire to compare the test trial performance for Shift and No Shift rats on a set of dependent measures that are typically taken as evidence for learning specific places in the Morris water task. Evaluation of the shift manipulation during a probe trial allowed for an assessment of persistence in searching (e.g., dwell, number of times the locations were visited) at each of the critical locations, which revealed that rats treat the relative spatial location in the pool much like they treat the absolute location when the pool is not shifted. As such, the movement vector hypothesis proposed here could also account for performance when the pool is not shifted as in the standard Morris water task. It is important to note this pattern of results and the conclusions that followed would not have been possible solely on the basis of evaluating the effects of manipulations during training.

The present results definitively refute the idea that rats swim to the escape platform exclusively by matching the current view of the distal cues to a perceptual memory of what the cues look like from the platform location (Wilkie \& Palfrey, 1987). If such a process operated, then the rats should have navigated to the absolute spatial location of the platform. The perceptual matching model presented by Wilkie and Palfrey (1987) also ignores the apparatus as a source of control. Although the pool wall does not disambiguate spatial locations, rats can learn to swim at the appropriate distance of the platform from the pool wall (Sutherland \& Rudy, 1988) and the present results indicate that this information can combine with direction information to control navigation and subsequent search behavior. Of importance, the present studies do not indicate that rats cannot learn the precise location of the platform or rule out the possibility that rats learn both the absolute spatial location of the platform and a directional response during training. Thus, a more conservative conclusion is that rats simply show a preference for directional responding over other types of responding when the pool is repositioned.
Although the results do not necessarily refute mapping theory, the idea that rats navigate in the water task on the basis of a global cognitive map is difficult to reconcile with the preference for directional responding and the evidence for control by the apparatus and distal cues described here. Directional responding as it has been described here is similar to the concept of orientation described by O'Keefe and Nadel (1978), which they argued to be distinct from a locale system involved in place navigation. Olton et al. (1979) contrasted place navigation and orientation by pointing out that a place would be analogous to a single point (e.g., in a Cartesian coordinate system), whereas orientation involves a vector (a line and direction). Thus, navigating by moving in a particular direction within the apparatus and room can be thought of as occurring along an axis, or line, whereas place navigation involves movement to a particular point. The present evidence for directional responding is clearly consistent with Olton's characterization of orientation and inconsistent with the idea that rats learn to navigate to a single point.

Given the dissociation of direction and place reported here, it is important for future studies to thoroughly evaluate the contribution of place information to water task learning. Whether rats are capable of learning to navigate to absolute locations or prefer place navigation in some situations could be addressed by moving the pool on each trial and keeping the platform at the same location or in the same direction, similar to the methods of Blodgett et al. (1949) and Skinner et al. (2003). If directional responding is the predominant form of learning in the water task and the contribution attributable to place information is negligible, then appealing to a system capable of learning precisely where reinforcement occurs to explain Morris water task performance would not be necessary. Rather, such demonstrations would underscore the importance of focusing on the behavioral processes involved in navigation between locations (Mackintosh, 2002; Shettleworth, 1998). That is not to say that explanations of navigation in terms of particular types of representations are not consistent with our observations. For example, one way in which the present results can be understood is to consider the space within the apparatus and the distal environment as two distinct frames of reference (Sutherland \& Hamilton, 2004). The results reported here would be expected if animals attempt to navigate to places within the apparatus reference frame on the basis of information about direction provided by the distal cue reference frame. Thus, rather than the operation of a global map-like representation, the results presented here are consistent with the operation of distinct reference frames (Gibson, 2001; Sutherland \& Hamilton, 2004) or separate, nonglobal maps (Cheng, 1986; Gallistel, 1990).

Recently, Knierim and colleagues (Knierim \& Rao, 2003; Yoganarasimha \& Knierim, 2005) described physiological data consistent with distinct room and apparatus reference frames. After preferred firing patterns of hippocampal place cells (O'Keefe \& Dostrovsky, 1971) and head direction cells in anterior thalamus (Taube, 1995; Taube \& Burton, 1995) were established, the openfield apparatus was moved in the room. During apparatus translation, the preferred firing location and direction of the respective cell types remained bound to the apparatus rather than to absolute spatial locations defined by the distal cue reference frame. Another property of hippocampal place cells with clear relevance for the present results comes from the observation that some place cells are active primarily when an animal approaches a location from a particular direction (McNaughton, Barnes, \& O’Keefe, 1983; Mul- 
ler, Bostock, Taube, \& Kubie, 1994). These directional firing properties have been observed when animals take direct trajectories along highly consistent or constrained paths or search at specific locations (Markus et al., 1995) but not when animals explore open fields (McNaughton et al., 1983; Gothard, Skaggs, Moore, \& McNaughton, 1996) or randomly search an environment (Markus et al., 1995). The behavior of well-trained rats in the water task is unambiguously direct and focused, even when the pool is repositioned; therefore, these electrophysiological correlates of navigation-related behavior fit well with the present findings. Hippocampal damage (Morris, Garrud, Rawlins, \& O'Keefe, 1982; Pearce, Roberts, \& Good, 1998; Sutherland, Kolb, \& Whishaw, 1982; Sutherland et al., 2001) and alteration of hippocampal physiology (Moser, Krobert, Moser, \& Morris, 1998; Steele \& Morris, 1999) cause profound impairments in the water task; however, to our knowledge, these deficits have only been reported for cases in which the pool always remains in the same location. Thus, one question motivated by the present findings concerns the degree to which directional responding and place navigation in the water task are affected by hippocampal damage. Hippocampal lesions must disrupt all processes capable of supporting direct trajectories to the platform, including directional responding. However, an unambiguous demonstration of impaired directional responding in the water task in a situation where direction and place are not confounded is needed. A recent study by Stringer, Martin, and Skinner (2005) demonstrated that hippocampal lesions disrupt directional learning as well as place learning in a dry land maze when the apparatus was repeatedly moved and reinforcement was either located in the same place or in the same direction within the room. The methods used by Stringer et al. (2005) could be easily adapted to the water task and seem well suited to address this question. A definitive demonstration that directional responding in the water task depends on the hippocampus would have considerable implications for explanations of the biological bases of spatial navigation.

In summary, the results of the present study demonstrate two important features of spatial learning and navigation in the Morris water task. First, the results indicate that rats can learn to navigate to a hidden or cued escape platform by moving in a particular direction relative to distal cues and swimming at the appropriate distance from the pool wall. We propose that these processes reflect the operation of a movement vector composed of direction and distance information provided by distal cues and the pool wall, respectively. Alternatives to this explanation, such as cognitive mapping or perceptual matching, propose systems in which animals can learn the precise place where reinforcement occurs. If the type of movement vector proposed here is sufficient to account for navigation in the Morris water task, then appeal to explanations based on such systems would be unnecessary. It is important to note, however, that processes involved in true place navigation and processes involved in the movement vector proposed here need not be mutually exclusive and may in fact be related. Second, the results demonstrate that multiple sources of control operate during a single trial. This is evident in the results of all three experiments in that the pool wall, distal cues, and cued platform all controlled behavior. The framework presented by Sutherland and Hamilton (2004) emphasized the idea that multiple constituent processes can operate serially, in parallel, or rapidly alternate when animals navigate from one place to another (see also McDonald \& White, 1994). Considered in this way, the results described here reflect the operation of at least two constituent processes in the hidden platform task but do not rule out other critical processes that were not investigated. Identification of the constituent processes that operate during a single navigation trial, the basic principles involved in their operation, and the situations in which they operate are critical for advancing and evaluating theories of navigation.

\section{References}

Blodgett, H. C., \& McCutchan, K. (1947). Place versus response learning in the simple T maze. Journal of Experimental Psychology, 37, 412422

Blodgett, H. C., McCutchan, K., \& Mathews, R. (1949). Spatial learning in the T-maze: The influence of direction, turn, and food location. Journal of Experimental Psychology, 39, 800-809.

Chang, Q., \& Gold, P. E. (2003). Intra-hippocampal lidocaine injections impair acquisition of a place task and facilitate acquisition of a response task in rats. Behavioural Brain Research, 144, 19-24.

Cheng, K. (1986). A purely geometric module in the rat's spatial representation. Cognition, 23, 149-178.

Chew, G. L., Sutherland, R. J., \& Whishaw, I. Q. (1989). Latent learning does not produce instantaneous transfer of place navigation: A rejoinder to Keith and McVety. Psychobiology, 17, 207-209.

Devan, B. D., Petri, H. L., Mishkin, M., Stouffer, E. M., Bowker, J. L., Yin, P.-B., et al. (2002). A room with a view and a polarizing cue: Individual differences in the stimulus control of place navigation and passive latent learning in the water maze. Neurobiology of Learning and Memory, 78, 79-99.

Gallagher, M., Burwell, R., \& Burchinal, M. (1993). Severity of spatiallearning impairment in aging-Development of a learning index for performance in the Morris water maze. Behavioral Neuroscience, 107, $618-626$.

Gallistel, C. R. (1990). The organization of learning. Cambridge, MA: MIT Press.

Gibson, B. M. (2001). Cognitive maps not used by humans (Homo sapiens) during a dynamic navigational task. Journal of Comparative Psychology, 115, 397-402.

Gothard, K. M., Skaggs, W. E., Moore, K. M., \& McNaughton, B. L. (1996). Binding of hippocampal CA1 neural activity to multiple reference frames in a landmark-based navigation task. Journal of Neuroscience, $16,823-835$

Hamilton, D. A., Driscoll, I., \& Sutherland, R. J. (2002). Human place learning in a virtual Morris water task: Some important constraints on the flexibility of place navigation. Behavioural Brain Research, 129, $159-170$.

Hamilton, D. A., Rosenfelt, C. S., \& Whishaw, I. Q. (2004). Sequential control of navigation by locale and taxon cues in the Morris water task. Behavioural Brain Research, 154, 385-397.

Hull, C. L. (1943). Principles of behavior: An introduction to behavior theory. New York: Appleton-Century-Crofts.

Keith, J. R., \& McVety, K. M. (1988). Latent place learning in a novel environment and the influences of prior training in rats. Psychobiology, 16, 146-151

Knierim, J. J., \& Rao, G. (2003). Distal landmarks and hippocampal place cells: Effects of relative translation versus rotation. Hippocampus, 13, 604-617.

Mackintosh, N. J. (2002). Do not ask whether they have a cognitive map, but how they find their way about. Psicologia, 23, 165-185.

Markus, E. J., Qin, Y. L., Leonard, B., Skaggs, W. E., McNaughton, B. L., \& Barnes, C. A. (1995). Interactions between location and task affect the spatial and directional firing of hippocampal neurons. Journal of $\mathrm{Neu}$ roscience, $15,7079-7094$.

McDonald, R. J., \& White, N. M. (1994). Parallel information-processing in the water maze: Evidence for independent memory-systems involving 
dorsal striatum and hippocampus. Behavioral and Neural Biology, 61, 260-270.

McNaughton, B. L., Barnes, C. A., \& O'Keefe, J. (1983). The contributions of position, direction, and velocity to single unit-activity in the hippocampus of freely-moving rats. Experimental Brain Research, 52, $41-49$.

Morris, R. G. M. (1981). Spatial localisation does not require the presence of local cues. Learning and Motivation, 12, 239-260.

Morris, R. G. M. (1984). Developments of a water-maze procedure for studying spatial-learning in the rat. Journal of Neuroscience Methods, $11,47-60$.

Morris, R. G. M., Garrud, P., Rawlins, J. N. P., \& O’Keefe, J. (1982, June). Place navigation impaired in rats with hippocampal damage. Nature, 297, 681-683.

Moser, E. I., Krobert, K. A., Moser, M. B., \& Morris, R. G. M. (1998, September). Impaired spatial learning after saturation of long-term potentiation. Science, 281, 2038-2042.

Muller, R. U., Bostock, E., Taube, J. S., \& Kubie, J. L. (1994). On the directional firing properties of hippocampal place cells. Journal of Neuroscience, 14, 7235-7251.

O'Keefe, J., \& Dostrovsky, J. (1971). The hippocampus as a spatial map: Preliminary evidence from unit activity in the freely-moving rat. Brain Research, 34, 171-175.

O'Keefe, J., \& Nadel, L. (1978). The hippocampus as a cognitive map. Oxford, England: Clarendon Press.

Olton, D. S., Becker, J. T., \& Handelmann, G. E. (1979). Hippocampus, space, and memory. Behavioral and Brain Sciences, 2, 313-322.

Packard, M. G., \& McGaugh, J. L. (1996). Inactivation of hippocampus or caudate nucleus with lidocaine differentially affects expression of place and response learning. Neurobiology of Learning and Memory, 65, 65-72.

Pearce, J. M., Roberts, A. D. L., \& Good, M. (1998, November). Hippocampal lesions disrupt navigation based on cognitive maps but not heading vectors. Nature, 396, 75-77.

Pearce, J. M., Roberts, A. D. L., Redhead, E. S., \& Prados, J. (2000). The influence of passive preexposure on escape from a Morris pool. Journal of Experimental Psychology: Animal Behavior Processes, 26, 186-195.

Redhead, E. S., Roberts, A., Good, M., \& Pearce, J. M. (1997). Interaction between piloting and beacon homing by rats in a swimming pool. Journal of Experimental Psychology: Animal Behavior Processes, 23, 340-350.

Shettleworth, S. J. (1998). Cognition, evolution, and behavior. New York: Oxford University Press.

Skinner, D. M., Etchegary, C. M., Ekert-Maret, E. C., Baker, C. J., Harley, C. W., Evans, J. H., et al. (2003). An analysis of response, direction, and place learning in an open field and $\mathrm{T}$ maze. Journal of Experimental Psychology: Animal Behavior Processes, 29, 3-13.

Steele, R. J., \& Morris, R. G. M. (1999). Delay-dependent impairment of a matching-to-place task with chronic and intrahippocampal infusion of the NMDA-antagonist D-AP5. Hippocampus, 9, 118-136.
Stringer, K. G., Martin, G. M., \& Skinner, D. A. (2005). The effects of hippocampal lesions on response, direction, and place learning in rats. Behavioral Neuroscience, 119, 946-952.

Sutherland, R. J., Chew, G. L., Baker, J. C., \& Linggard, R. C. (1987). Some limitations on the use of distal cues in place navigation by rats. Psychobiology, 15, 48-57.

Sutherland, R. J., \& Dyck, R. H. (1984). Place navigation by rats in a swimming pool. Canadian Journal of Psychology, 38, 322-347.

Sutherland, R. J., \& Hamilton, D. A. (2004). Rodent spatial navigation: At the crossroads of cognition and movement. Neuroscience Biobehavioral Reviews, 28, 687-697.

Sutherland, R. J., Kolb, B., \& Whishaw, I. Q. (1982). Spatial mapping: Definitive disruption by hippocampal or frontal cortical damage in the rat. Neuroscience Letters, 31, 271-276.

Sutherland, R. J., \& Linggard, R. (1982). Being there: A novel demonstration of latent spatial-learning in the rat. Behavioral and Neural Biology, 36, 103-107.

Sutherland, R. J., \& Rudy, J. W. (1988). Place learning in the Morris place navigation task is impaired by damage to the hippocampal-formation even if the temporal demands are reduced. Psychobiology, 16, 157-163.

Sutherland, R. J., Weisend, M. P., Mumby, D., Astur, R. S., Hanlon, F. M., Koerner, A., et al. (2001). Retrograde amnesia after hippocampal damage: Recent vs. remote memories in two tasks. Hippocampus, 11, 27-42.

Taube, J. S. (1995). Head direction cells recorded in the anterior thalamic nuclei of freely moving rats. Journal of Neuroscience, 15, 70-86.

Taube, J. S., \& Burton, H. L. (1995). Head direction cell-activity monitored in a novel environment and during a cue conflict situation. Journal of Neurophysiology, 74, 1953-1971.

Tolman, E. C. (1948). Cognitive maps in rats and men. Psychological Review, 55, 189-209.

Tolman, E. C., Ritchie, B. F., \& Kalish, D. (1946). Studies in spatial learning. II. Place learning versus response learning. Journal of Experimental Psychology, 3, 221-229.

Weisend, M. P., Klein, R. L., Hoesing, J. M., Astur, R. S., Koerner, A., McDonald, R. J., et al. (1995). Morris water task: Which cues define locations? Society for Neuroscience Abstracts, 21, 1939-1939.

Whishaw, I. Q., Mittelman, G., Bunch, S. T., \& Dunnett, S. B. (1987). Impairments in the acquisition, retention and selection of spatial navigation strategies after medial caudate-putamen lesions in rats. Behavioural Brain Research, 24, 125-138.

Wilkie, D. M., \& Palfrey, R. (1987). A computer simulation model of rats' place navigation in the Morris water maze. Behavior Research Methods Instruments and Computers, 19, 400-403.

Yoganarasimha, D., \& Knierim, J. J. (2005). Coupling between place cells and head direction cells during relative translations and rotations of distal landmarks. Experimental Brain Research, 160, 344-359.

Received July 11, 2006

Revision received January 5, 2007

Accepted January 9, 2007 